\section{A prospective randomized trial of the optimal dose of mannitol for intraoperative brain relaxation in patients undergoing craniotomy for supratentorial brain tumor resection}

TO THE READERSHIP: An error appeared in the article by Seo et al. (Seo H, Kim E, Jung H, et al: A prospective randomized trial of the optimal dose of mannitol for intraoperative brain relaxation in patients undergoing craniotomy for supratentorial brain tumor resection. J Neurosurg 126:1839-1846, 2017).
Several ratios were incorrectly calculated in Tables 1 and 2. The ratios should be $2(6.5 \%), 3(9.7 \%)$, and 6 (19.4\%) - not 2 (6.4\%), 3 (9.6\%), and 6 (19.2\%). The updated tables are shown here.

The article has been corrected online as of June 1,2018.

Hee-Pyoung Park, MD, PhD Seoul National University College of Medicine, Bundang-gu, Seongham, Korea

\section{INCLUDE WHEN CITING}

Published online June 1, 2018; DOI: 10.3171/2018.1.JNS16537a.

CAANS 2018, except where prohibited by US copyright law

TABLE 1. Demographics in the 124 patients included in this study

\begin{tabular}{|c|c|c|c|c|c|}
\hline Variables & Group A $(n=31)$ & Group B $(n=31)$ & Group C ( $n=31)$ & Group D (n= 31) & $p$ Value \\
\hline Mean age in yrs (range) & $52.5(32-74)$ & $53.1(28-78)$ & $52.1(23-79)$ & $48.9(24-76)$ & 0.474 \\
\hline Male sex & $20(64.5 \%)$ & $15(48.4 \%)$ & $12(38.7 \%)$ & $14(45.2 \%)$ & 0.214 \\
\hline Mean height, $\mathrm{cm}$ & $165.5(9.4)$ & $162.7(8.9)$ & $162.4(7.9)$ & $163.2(9.5)$ & 0.508 \\
\hline Mean weight, $\mathrm{kg}$ & $65.0(10.0)$ & $65.7(12.8)$ & $63.6(10.0)$ & $64.0(13.0)$ & 0.723 \\
\hline Mean BMI, $\mathrm{kg} / \mathrm{m}^{2}$ & $23.5(2.5)$ & $24.9(4.4)$ & $23.9(3.9)$ & $23.9(3.9)$ & 0.447 \\
\hline Tumor type & & & & & 0.868 \\
\hline Anaplastic astrocytoma & $0(0 \%)$ & $2(6.5 \%)$ & $1(3.2 \%)$ & $4(12.9 \%)$ & \\
\hline Glioblastoma & $5(16.1 \%)$ & $6(19.4 \%)$ & $5(16.1 \%)$ & $6(19.4 \%)$ & \\
\hline Oligodendroglioma & $1(3.2 \%)$ & $3(9.7 \%)$ & $2(6.5 \%)$ & $1(3.2 \%)$ & \\
\hline Other glioma & $3(9.7 \%)$ & $3(9.7 \%)$ & $1(3.2 \%)$ & $3(9.7 \%)$ & \\
\hline Meningioma & $16(51.6 \%)$ & $10(32.2 \%)$ & $14(45.2 \%)$ & $12(38.7 \%)$ & \\
\hline Metastasis & $3(9.7 \%)$ & $5(16.1 \%)$ & $4(12.9 \%)$ & $2(6.5 \%)$ & \\
\hline Other & $3(9.7 \%)$ & $2(6.5 \%)$ & $4(12.9 \%)$ & $3(9.7 \%)$ & \\
\hline Tumor location & & & & & 0.932 \\
\hline Frontal & $21(67.7 \%)$ & $16(51.6 \%)$ & $16(51.6 \%)$ & $18(58.1 \%)$ & \\
\hline Temporal & $4(12.9 \%)$ & $4(12.9 \%)$ & $6(19.4 \%)$ & $3(9.7 \%)$ & \\
\hline Parietal & $2(6.5 \%)$ & $5(16.1 \%)$ & $4(12.9 \%)$ & $4(12.9 \%)$ & \\
\hline Occipital & $1(3.2 \%)$ & $1(3.2 \%)$ & $2(6.5 \%)$ & $2(6.5 \%)$ & \\
\hline Sphenoidal & $1(3.2 \%)$ & $3(9.7 \%)$ & $1(3.2 \%)$ & $1(3.2 \%)$ & \\
\hline Other & $2(6.5 \%)$ & $2(6.5 \%)$ & $2(6.5 \%)$ & $3(9.7 \%)$ & \\
\hline Mean max tumor diameter, $\mathrm{mm}$ & $50.6(14.4)$ & $45.5(15.4)$ & $50.0(16.2)$ & $50.7(11.8)$ & 0.476 \\
\hline Mean midline shift, mm & $9.2(4.3)$ & $10.0(6.7)$ & $8.4(5.9)$ & $9.0(4.0)$ & 0.695 \\
\hline Peritumoral edema $>10 \mathrm{~mm}$ & $24(77.4 \%)$ & $25(80.6 \%)$ & $25(80.6 \%)$ & $27(87.1 \%)$ & 0.879 \\
\hline
\end{tabular}

The administered dose of mannitol is $0.25 \mathrm{~g} / \mathrm{kg}$ in Group A, $0.5 \mathrm{~g} / \mathrm{kg}$ in Group B, $1.0 \mathrm{~g} / \mathrm{kg}$ in Group C, and $1.5 \mathrm{~g} / \mathrm{kg}$ in Group D. Values are presented as the number of patients (\%) unless indicated otherwise. Mean values are presented as the mean (SD) unless indicated otherwise. 
TABLE 2. Intraoperative data and postoperative outcomes

\begin{tabular}{|c|c|c|c|c|}
\hline Variables & Group A ( $n=31)$ & Group B (n = 31) & Group C ( $n=31)$ & Group D ( $n=31)$ \\
\hline \multicolumn{5}{|l|}{ Intraop data } \\
\hline $\begin{array}{l}\text { Mean time to assess brain relaxation from } \\
\text { end of mannitol administration, mins }\end{array}$ & $33(5)$ & $32(6)$ & $32(5)$ & $35(6)$ \\
\hline Median op time, mins & 269 [199-341] & 258 [239-377] & 263 [235-349] & $323[235-391]$ \\
\hline Median anesthesia time, mins & 330 [294-415] & $330[320-458]$ & $340[305-420]$ & $390[300-474]$ \\
\hline Median administered fluids, $\mathrm{ml}$ & 2250 [1575-2825] & $1800[1700-2400]$ & $2300[1500-2850]$ & 2225 [1575-2913] \\
\hline Median total urine output, $\mathrm{ml}$ & 1540 [725-1908] & 1145 [910-1545] & $1500[1180-2500] \dagger$ & $2195[1394-3005] \dagger, \ddagger$ \\
\hline Median total estimated blood loss, ml & $600[300-800]$ & $540[288-850]$ & $500[350-700]$ & $650[400-1100]$ \\
\hline Median total fluid balance, $\mathrm{ml}$ & 520 [28-912] & $330[-40$ to 833$]$ & $-70[-300$ to 945$]$ & $-175[-670 \text { to } 620]^{* *}$ \\
\hline \multicolumn{5}{|l|}{$\begin{array}{l}\text { Median serum osmolality after mannitol } \\
\text { administration, } \mathrm{mOsm} / \mathrm{kg}\end{array}$} \\
\hline At T1 & 299 [295-302] & 298 [295-304] & 295 [291-300] & 299 [291-303] \\
\hline At T2 & 303 [301-309] & $303[300-307]$ & $306[303-310]^{\dagger}$ & $315[310-317]^{\star}, \dagger, \ddagger$ \\
\hline At T3 & 303 [299-308] & 303 [299-306] & $306[302-310]^{\dagger \dagger}$ & $312[309-314]^{*}, \dagger, \ddagger$ \\
\hline At T4 & $303[300-306]$ & 299 [295-307] & 305 [299-309] & $307[304-313]^{* *}$, ,†,拉 \\
\hline \multicolumn{5}{|l|}{ Additional methods for brain relaxation } \\
\hline Overall & $6(19.4 \%)$ & $7(22.6 \%)$ & $3(9.7 \%)$ & $2(6.5 \%)$ \\
\hline Additional mannitol administration & $4(12.9 \%)$ & $0(0 \%)$ & $0(0 \%)$ & $0(0 \%)$ \\
\hline Transient hyperventilation & $1(3.2 \%)$ & $2(6.5 \%)$ & $2(6.5 \%)$ & $2(6.5 \%)$ \\
\hline $\begin{array}{l}\text { Transient hyperventilation + additional man- } \\
\text { nitol administration }\end{array}$ & $1(3.2 \%)$ & $4(12.9 \%)$ & $1(3.2 \%)$ & $0(0 \%)$ \\
\hline Reverse Trendelenburg position & $0(0 \%)$ & $1(3.2 \%)$ & $0(0 \%)$ & $0(0 \%)$ \\
\hline \multicolumn{5}{|l|}{ Postop outcome } \\
\hline Median ICU stay, days & $2[2-3]$ & $2[2-3]$ & $2[2-4]$ & $3[2-5]$ \\
\hline Median hospital stay, days & 9 [8-13] & $9[8-13]$ & $12[9-16]$ & $11[8-20]$ \\
\hline
\end{tabular}

$\mathrm{T} 1$ = just before the mannitol administration; $\mathrm{T} 2=30$ minutes after the end of mannitol administration; $\mathrm{T} 3=60$ minutes after the end of mannitol administration; $\mathrm{T} 4=180$ minutes after the end of mannitol administration.

Values are presented as the number of patients (\%) unless indicated otherwise. Mean values are presented as the mean (SD). Median values are presented as the median [IQR].

${ }^{*} p<0.01$, compared with Group A; ${ }^{* *} p<0.05$, compared with Group A; $\nmid p<0.01$, compared with Group B; $\uparrow \nmid p<0.05$, compared with Group B; $\ddagger p<0.01$, compared with Group C; $\ddagger \ddagger p<0.05$, compared with Group C. 